\title{
Il s'agit du financement, non des compétences
}

\author{
En lien avec l'initiative parlementaire «Pour la reconnaissance légale de la responsa- \\ bilité infirmière», malentendus et insinuations - usuels, semble-t-il, en politique - \\ ont fini par créer une certaine confusion. Une clarification s'impose, notamment \\ sur le plan juridique.
}

Pierre-André Wagner

Avocat, LL.M., infirmier diplômé SG, responsable du service juridique de l'Association suisse des infirmières et infirmiers (ASI)

Un commentaire de la FMH se trouve à la page 1097. pierre-andre.wagner[at] sbk-asi.ch
La première question qui suscite la confusion est celle-ci: l'initiative modifiera-t-elle les limites entre pratique médicale et pratique infirmière? Et le corollaire: les infirmières se verront-elles attribuer des compétences qui étaient jusque-là du ressort des médecins - avec des conséquences imprévisibles en termes de responsabilité légale?

La réponse est «non»- et «non». Car cette initiative porte sur la LAMal - une loi qui ne règle pas les questions de responsabilité.

L'initiative apporte toutefois - sur le plan de la responsabilité aussi - une clarification longtemps attendue, dont bénéficient tant les médecins que les infirmières.

En d'autres termes, l'initiative vise tout simplement à ce que les prestations que les infirmières fournissent déjà maintenant sous leur propre responsabilité (légale et professionnelle), c'est-à-dire sans mandat ou visa médical, soient également prises en charge par l'assurance-maladie sans être visées par un médecin.

\section{Une image erronée des soins}

Le titre peu élégant de l'initiative a pourtant été soigneusement choisi: il ne s'agit pas d'une valorisation de la profession infirmière, mais de la reconnaissance de sa responsabilité. On ne peut reconnaître que ce qui existe déjà. L'initiative demande que la responsabilité que portent - maintenant déjà - les infirmières soit enfin reconnue dans la LAMal.

Répétons que la LAMal ne règle pas de compétences. Elle ne dit pas quelles professions de la santé sont responsables de quoi et qui doit ou peut faire quoi. Ce sont les lois cantonales de la santé qui déterminent les tâches et les responsabilités des infirmières. La LAMal réglemente uniquement le financement. Cette loi fédérale définit quelles sont les prestations que les caisses-maladie doivent rembourser à quel prestataire et à quelles conditions.

La clef pour comprendre cette initiative réside dans une particularité de la profession infirmière que la plupart des non-professionnels - y compris la plupart des politiciens - ignorent: à savoir le fait que les soins infirmiers comportent deux domaines. Le premier comprend les actes diagnostiques et thérapeutiques des infirmières. Ces actes faisant partie des compétences-clefs des médecins, les infirmières agissent ici sur délégation exclusivement. Des stéréotypes indécrottables réduisent les soins infirmiers à ce domaine - l'infirmière comme l'auxiliaire du médecin - une représentation faussée qui se retrouve malheureusement dans la LAMal.

\section{Des médecins dépassés}

On en oublie alors le second domaine de l'art infirmier, dans lequel les professionnelles travaillent de manière indépendante, autonome et surtout: sous leur propre responsabilité. Ce domaine, dont font partie l'évaluation des besoins en soins, les conseils au patient et à ses proches et bien d'autres choses, est piloté par le processus de soins et n'a pas le moindre point commun avec le domaine de compétences des médecins. Les bases légales du domaine autonome des soins infirmiers se trouvent dans les lois cantonales sur la santé. A titre d'exemple, un extrait de la Loi vaudoise sur la santé publique du 29 mai 1985, art. 124 al. 1 et 2:

«1. L'infirmière est une personne formée pour donner professionnellement les soins ci-après:

a) soutien et suppléance dans les activités de la vie quotidienne;

b) accompagnement dans les situations de crise et dans la période de fin de vie;

c) participation aux mesures préventives, diagnostiques et thérapeutiques;

d) participation à des actions de prévention des maladies et des accidents ainsi que de maintien et de promotion de la santé, de réadaptation fonctionnelle et de réinsertion sociale;

e) contribution à l'amélioration de la qualité et de l'efficacité des soins infirmiers, au développement de la profession et collaboration à des programmes de recherche concernant la santé publique.

2. L'infirmière donne ces soins de façon autonome à l'exception de la lettre c) où elle agit sur délégation du médecin.»

La LAMal est donc diamétralement en contradiction avec les lois cantonales. Ainsi, les infirmières disposent indéniablement d'un domaine autonome, mais les prestations relatives à ce domaine ne sont 
remboursées par les caisses-maladie... que s'il y a prescription médicale.

Comment expliquer cette anomalie - et quels risques présente-t-elle au niveau de la responsabilité légale? Cette aberration législative est issue d'une part des clichés déjà évoqués au sujet de la profession infirmière, et d'autre part d'une représentation pas moins naïve du médecin en tant que "gatekeeper», sorte de juge impartial et détenteur du savoir concernant les prestations infirmières nécessaires. Il se voit contraint de valider des prestations pour lesquelles il n'est pas compétent - justement parce qu'elles font partie du domaine de responsabilité propre d'une autre profession. Avec sa notion d' «auxiliaire autonome», la législation en vigueur crée une confusion fatale.

\section{Qui est responsable de quoi?}

Commençons par le domaine des actes effectués sur délégation médicale: la responsabilité civile, pénale et disciplinaire incombe totalement à l'infirmière pour les actes qui lui ont été délégués en conformité avec les principes juridiques de la délégation. Si elle commet une faute, maintenant déjà, elle assume seule la responsabilité [1], indépendamment de la prescription ou signature du médecin.

Cela vaut bien évidemment d'autant plus pour le domaine de compétences propre de l'infirmière, assumé sous sa propre responsabilité. Par la signature exigée par la LAMal, le médecin n'aura à répondre d'aucune responsabilité civile, pénale ou disciplinaire pour l'exécution de cette prestation.

Cette signature n'a pour seul et unique but que le déclenchement d'un remboursement de l'assurance-maladie: le médecin se porte garant que la prestation répond aux exigences de la loi sur l'assurance-maladie - ce qu'en réalité il ne peut pas, pour les raisons déjà évoquées.

\section{Une aberration coûteuse}

La pratique quotidienne des infirmières indépendantes montre à quel point tout ceci est absurde

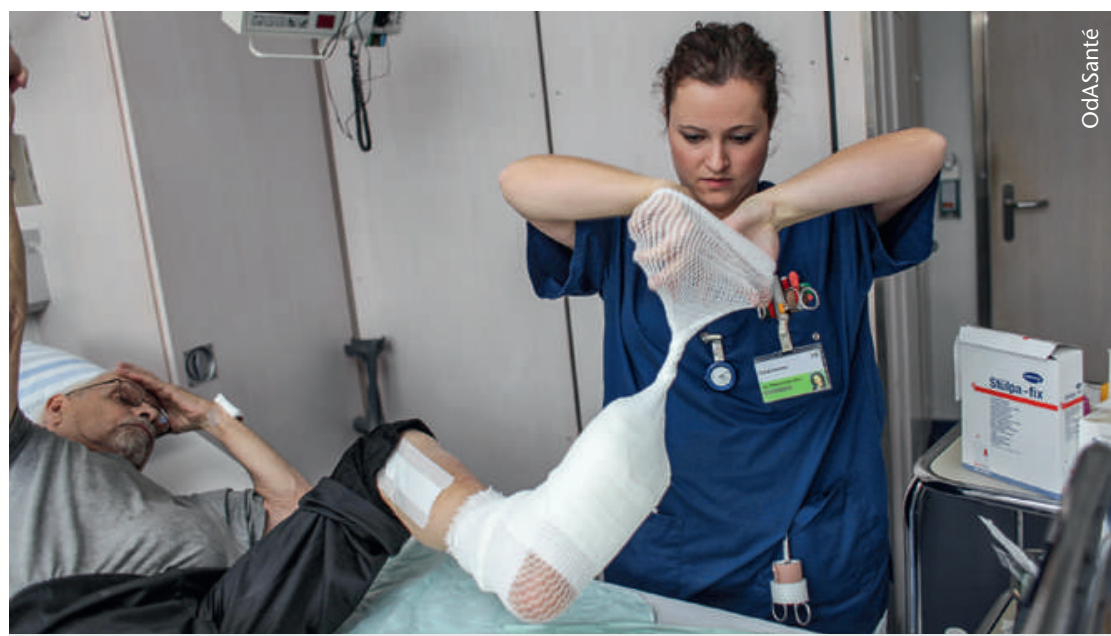

Déjà maintenant les infirmières fournissent des prestations sous leur propre responsabilité légale et professionnelle. puisque c'est souvent exactement l'inverse qui se produit: on attend en effet d'elles, et non du médecin prescripteur, de démontrer l'économicité, l'utilité et l'efficacité des mesures de soins thérapeutiques!

Que la signature requise du médecin pour les mesures de soins effectuées sous la responsabilité de l'infirmière représente une aberration (coûteuse), les caisses-maladie avisées l'ont compris [2], les médecins avisés également [3] - car rien n'est plus toxique et décourageant pour la collaboration interprofessionnelle qu'une réglementation des compétences qui ne coïncide pas avec celle des responsabilités [4]. L'initiative parlementaire Joder ne demande que de mettre en accord la responsabilité des infirmières avec les compétences de ces dernières - et d'ajuster la loi à la réalité de la profession - ni plus ni moins.

\section{Acheter chat en poche?}

Une autre question source d'angoisses est la suivante: est-on bien au clair sur les activités qui relèvent du domaine autonome des soins et celles qui relèvent du domaine médico-délégué, ce d'autant plus que l'initiative stipule explicitement que cette délimitation incombe au Conseil fédéral? La réponse, encore une fois, s'impose: oui, on ne peut plus clair. L'art. 7 de l'Ordonnance sur les prestations de la LAMal [5], qui définit les prestations de soins à la charge de l'assurance de base, distingue clairement entre les mesures diagnostiques et thérapeutiques (énumérées d'ailleurs de manière exhaustive) d'une part, et les mesures d'évaluation, de conseil, de coordination et des soins de base d'autre part.

La crainte d'acheter chat en poche en soutenant l'initiative n'est pas justifiée. La simple référence à la systématique de la LAMal suffit pour dissiper tout doute: les prestations assurées sont, par principe, décrites et énumérées par voie d'ordonnance. On peut difficilement reprocher à l'initiative de s'en tenir au système actuel de la LAMal.

\section{Références}

1 Elle pourrait se décharger uniquement en affirmant que le médecin déléguant aurait été à l'encontre des principes de délégation, par ex. en donnant des instructions insuffisantes ou en ayant négligé son devoir de surveillance et de contrôle, respectivement en affirmant qu'en raison de sa formation ou de son expérience, elle n'était pas en mesure d'évaluer correctement les risques de l'activité déléguée.

2 V. l'exposé d'Annette Jamieson (politique de la santé Helsana) à l'occasion de la rencontre du groupe parlementaire Soins infirmiers du 12 mars 2014.

3 V. lettre de lecteur du Dr Severin Lüscher dans le Bulletin des médecins suisses 2014/11 au sujet de l'éditorial de Jürg Schlup, président de la FMH «Oui au renforcement de l'interprofessionnalité - non à l'initiative» dans l'édition 8/2014.

4 Wagner PA. Interdisziplinäre Kooperation zwischen Ethik und Recht. In: Settimio Monteverde (Ed.). Handbuch Pflegeethik. Stuttgart; 2012. p. 74 ss.

5 Ordonnance du DFI du 29 septembre 1995 sur les prestations de l'assurance-maladie obligatoire (OPAS), 832.112.31. 